\title{
Longer Diagnosis-surgery Duration Is Associated With an Increased Risk of Early Postoperative Complications for Crohn's Disease: A Retrospective Study
}

\section{Xuanyi Chen}

Renji Hospital

Siqi Zhang

Renji Hospital

Fanru Shen

Renji Hospital

Yuan Shi

Renji Hospital

Sailiang Liu

Renji Hospital

Yihua Jin

Renji Hospital

Ming Zhong

Renji Hospital

\section{Yuqi Qiao}

Renji Hospital

Minhao Yu ( $\square$ fishmeangood@163.com )

Renji Hospital

\section{Research Article}

Keywords: Crohn's disease, diagnosis-surgery duration, postoperative complications, risk factors, retrospective study

Posted Date: December 20th, 2021

DOI: https://doi.org/10.21203/rs.3.rs-1142302/v1

License: (9) (1) This work is licensed under a Creative Commons Attribution 4.0 International License. Read Full License 


\section{Abstract}

Background: Early postoperative complications(ePOCs) frequently occur in Crohn's patients after surgery. The risk factors of ePOCs for Crohn's disease (CD), however, remain controversial. We aimed to assess the incidence and risk factors of ePOCs in CD patients after surgical resection.

Methods: The retrospective study was conducted on 97 patients undergoing surgeries between January 2010 and September 2019 for Crohn's disease in a tertiary hospital in China.

Results: In total, 33 patients (34.0\%) experienced ePOCs, including 11 intra-abdominal septic complications (11.3\%) and 1 postoperative death (1.0\%). Severe complications (Dindo-Clavien III-IV) were seen in 8 patients (8.2\%). In multivariate analysis, diagnosis-surgery duration exceeding 6 months(odds-ratio [OR] $=4.07$; confidence interval [CI] 95\%[1.10-15.09], $\mathrm{P}=0.036$ ), serum platelet count $<300 * 1000 / \mathrm{mm}^{3}$ (odds-ratio [OR] $=6.74$; confidence interval [CI] 95\%[1.58-28.71], $\mathrm{P}=0.01$ ) and serum gamma-glutamyl transpeptidase(GGT) level $>10 \mathrm{U} / \mathrm{L}$ (odds-ratio [OR]=9.22; confidence interval [CI] 95\% [1.23-68.99], $P=0.031)$ were identified as independent risk factors for ePOCs. Preoperative exposure to anti-tumor necrosis factor (TNF) agents $(P=1.00)$ were not associated with a higher risk of ePOCs. $34.0 \%$ of $\mathrm{CD}$ patients developed ePOCs after surgical resection.

Conclusions: Diagnosis-surgery duration exceeding 6 months, serum platelet count $<300 * 1000 / \mathrm{mm}^{3}$, and serum GGT level $>10 U / L$ were associated with an increased risk of ePOCs. Preoperative exposure to antiTNF agents were not associated with a higher risk of ePOCs.

\section{Introduction}

Crohn's disease is an immune-mediated inflammatory bowel disease characterized by chronic skip lesions that can affect any part of the gastrointestinal tract, leading to bowel damage and disability ${ }^{1}$. As a global disease, its worldwide incidence and prevalence are steadily increasing with time ${ }^{2}$. Since the 1980s, the introduction of biological therapies such as monoclonal antibody against tumor necrosis factor (TNF) and vedolizumab changed the paradigm of treatment, leading to improved response and remission in patients ${ }^{3-5}$. Though the risk of surgery in patients with Crohn's disease shows a decreasing trend thanks to advances in pharmacotherapy, about $50 \%$ of patients develop complications refractory to medical therapy and will still require surgery within 10 years after diagnosis ${ }^{6}$.

According to the literature, early postoperative complications(ePOCs) could occur in up to $37.5 \%$ of $C D$ patients after surgery ${ }^{7-13}$. Therefore, numerous studies have tried to explore the risk factors related to ePOCs which include low albumin level, penetrating disease, laparoscopic approach, preoperative exposure to steroids ${ }^{7-13}$. On the one hand, data remain conflicting regarding on some variables such as preoperative use of anti-TNF agents ${ }^{8-13}$; on the other hand, many variables are not included in the preexisting studies such as the diagnosis-surgery duration and some preoperative biological data. 
Therefore, better knowledge of risk factors for ePOCs of CD would help to define a high-risk population where prevention for postoperative complications would be beneficial.

The purpose of this study was to reliably identify incidence and risk factors of ePOCs after surgery for CD in a retrospective cohort from a high-volume tertiary center to improve postoperative outcomes.

\section{Methods}

\subsection{Patient selection}

This was a single-center retrospective study based on a database of 97 patients who underwent surgical resection for primary or recurrent Crohn's disease between January 2010 and September 2019. Patients pathologically diagnosed with Crohn's disease intraoperatively were included. Exclusion criteria were surgeries without pathological examination such as perianal surgery and abscess drainage alone, and missing or invalid data precluding analysis. 97 patients were eligible for analysis after these exclusion criteria were applied. This study was approved by Renji hospital's research and ethics committee (KY2019-180).

\subsection{Data collection}

Data were retrieved in a standardized format by expert gastroenterologists from Renji hospital medical records. Demographic data (age, sex, age at diagnosis, diagnosis-surgery duration), clinical data (disease location and behavior according to the Montreal classification ${ }^{14}$, presence of perianal manifestation and multiple lesions, history of intestinal resection and anal fistula surgery, and the indications for surgery), data on medication (steroids, IFX, 5-ASA, MTX, Azathioprine, Tacrolimus use within 2 months before surgery and their use history), preoperative laboratory testing (including serum hemoglobin, leukocyte, platelets, C-reactive protein, erythrocyte sedimentation rate, albumin, prealbumin, alanine transaminase, glutamic-oxalacetic transaminase, gamma-glutamyl transpeptidase, direct bilirubin, total bilirubin, urea, creatinine, uric acid, $\mathrm{Na}$ ion, $\mathrm{K}$ ion, procalcitonin levels), and operative data (emergency surgery, surgical approach, anastomotic configuration, length of hospital stay) were collected. All operations were performed by specialized surgeons from colorectal surgery department in Renji Hospital. And the surgical techniques (including surgery approach, anastomosis vs. stoma, etc.) were decided on a per-patient basis and left to the discretion of the surgeon based on patient's disease characteristics and intra-operative findings.

\subsection{Evaluated outcomes}

Surgical outcome was determined by postoperative complications and follow-up. Early postoperative complications(ePOCs) were defined as any deviation from the normal postoperative course within 30 days after surgery, and classified into three categories; (i) intra-abdominal septic complications (IASC) (including abdominal abscess and anastomotic leak confirmed radiologically), (ii)extra-abdominal septic complications(including wound infection, pneumonia and septic shock) and (iii)non-septic complications(including enterocutaneous fistula, hemorrhage, bowel obstruction, gastric retention, 
thrombosis, malnutrition, anemia). Specifically, abdominal abscess was defined as an abdominal mass or an area of localized abdominal tenderness in a feverish patient, confirmed by radiological evidence; bowel obstruction was defined as bowel dysfunction associated with clinical manifestations such as abdominal distention or vomiting. Data on aforementioned ePOCs were collected in a standardized format by appropriate laboratory tests and/or imaging modalities and were reviewed in detail. The severity of ePOCs was graded as I, II, III, IV, or V, according to the Dindo-Clavien classification ${ }^{15}$. Grades I, II were considered to be minor complications while grades III-V were considered to be major complications. Furthermore, the length of hospital stay was also noted.

\subsection{Statistical analysis}

The SPSS program version 21 was used for data analysis. Categorical variables were represented as the number (percentage). The primary endpoint was the occurrence of ePOCs. Normality of distribution for quantitative variables was evaluated using the Kolmogorov-Smirnov tests. Normally distributed variables were described as mean value ( \pm standard deviation), whereas non-normally distributed ones were presented as median (25th -75 th interquartile range [IQR]). In order to identify the risk factors of ePOCs, univariate and multivariate analyses were performed to examine the relationship between the occurrence of ePOCs and variables related to the patient characteristics and their clinical parameters. Univariate analysis was conducted using Pearson's chi-square test or Fisher's exact test to compare categorical variables, as appropriate. All variables associated with $P$ values $<0.1$ were included in a binary logistic regression model, expressed as the odds ratio (OR) (95\% confidence interval (Cl)). All P-values were 2sided and P-values $<0.05$ were considered statistically significant.

\section{Results}

\subsection{Demographic and clinical characteristics}

From January 2010 and September 2019, 97 patients underwent surgeries for CD. Their demographic and clinical characteristics at the time of surgery are summarized in Table 1. Our population had a median age of 43 years (IQR 37-52 years), and included 64 male patients (66.0\%). According to Montreal classification, the majority of patients $(n=57,58.8 \%)$ were diagnosed with $C D$ between $17-40$ years old(A2); 34 patients (35.1\%) presented with disease localized in ileum(L1) and 26 patients (26.8\%) had colon(L2) involvement only; clinical disease behavior was non- stricturing/non-penetrating in 22 patients $(22.7 \%)$, stricturing in 41 patients $(42.3 \%)$ and penetrating in 34 patients $(35.0 \%)$. Perianal lesion was detected in $27(27.8 \%)$ cases. $15(15.5 \%)$ patients had a history of abdominal surgery. $13(13.4 \%)$ patients had previously undergone anal fistula surgery.

\subsection{Medications}

The medication history and preoperative (within 2 months before surgery) medical treatment are specified in Table 1. For patients' medication history, 34 (35.1\%) patients had been treated with steroids, including either systemic steroids or budesonide. 41 (42.3\%) patients had received 5-aminosalicylic acid, 
and 23 (23.7\%) patients had received azathioprine in their medical records; fewer patients had been treated with infliximab $(8,8.2 \%)$, methotrexate $(2,2.1 \%)$, tacrolimus $(3,3.1 \%)$. As for preoperative medical treatment, 19 patients had received steroids within 2 months before surgery, and 26 (26.8\%) patients had been treated with 5-aminosalicylic acid (with 1 on infliximab, 9 on azathioprine and 1 on tacrolimus).

\subsection{Preoperative laboratory testing}

Laboratory tests at the time of surgery are detailed in Table 1. In the routine blood tests, the median leukocyte count was $7390 / \mathrm{mm} 3$ (5100-12500), the mean hemoglobin level was $11.2 \mathrm{~g} / \mathrm{dl}$, and the median platelet count was $245 \times 109 / \mathrm{L}$ (195-341). The median C-reactive protein level at surgery was 6.38 (1.73-21.30) $\mathrm{mg} / \mathrm{dl}$ while the median ESR level was $17(10.25-37.5) \mathrm{g} / \mathrm{dl}$, and the median prealbumin level was $155.85 \mathrm{mg} / \mathrm{L}$ (105.73-221.75). A minority of patients $(n=19,19.6 \%)$ had poor preoperative nutritional status defined as preoperative serum albumin $<30 \mathrm{~g} / \mathrm{dL}$. For liver function related index, the median serum level of ALT, AST, and GGT was 11(7.0-18.3), 14(11.0-17.25), and 17.3(10.45-32.25) U/L, respectively (3.9 and $9.6 \mathrm{umol} / \mathrm{L}$ on median serum direct and total bilirubin). For renal function test, the median serum creatine and uric acid level was 59.1(47.5-71) and 216.45(96.49), respectively. Data regarding serum procalcitonin level were only collected in 28 cases, most of which were $>0.02 \mathrm{ng} / \mathrm{ml}$ $(\mathrm{n}=22,75.9 \%)$.

\subsection{Surgical data}

The median duration from diagnosis to surgery was 4(0-25.5) months, and more than $41.2 \%$ patients did not undergo surgery until 6 months after diagnosis. Emergency surgery was performed in $23(23.7 \%)$ cases; laparoscopic surgery in $43(44.3 \%)$ patients, and anastomosis was preferred in a majority of patients $(n=90,92.8 \%)$. The indications for surgery were obstruction in $40(41.2 \%)$, fistula in $10(10.3 \%)$, perforation in $18(18.6 \%)$, bleeding in $6(6.2 \%)$ and medication-refractory disease in $5(5.2 \%)$ cases. The median postoperative hospital stay was 10 days $(7-13)$.

\section{4 ePOCs}

Overall, 42 ePOCs were observed in 33 patients (34.0\%), including 25(25.8\%) minor complications (DindoClavien grade I/II) and 8(8.2\%) major complications (Dindo-Clavien grade III-V) (Table 2). Multiple complications occurred in 6 patients $(6.2 \%)$. Among all the ePOCs, wound infection was the most common ePOC $(n=13,13.4 \%)$. IASCs were recorded in 11 patients $(11.3 \%)$, including abdominal abscess $(n=9,9.3 \%)$ and anastomotic leak $(n=2,2.1 \%)$. Extra-Abdominal septic complications occurred in 19 patients (19.6\%). Bowel obstruction was the most frequent non-septic complication (5.2\%), while postoperative hemorrhage and malnutrition were recorded in 1 patient (1.0\%). Other non-septic complications included enterocutaneous fistula (1.0\%), gastric retention (2.1\%), and anemia (1.0\%). No thromboembolic complications were observed. One patient died during the 30-day postoperative period. The median length of postoperative hospital stay was 10 days (7-13), which is significantly correlated with the occurrence of postoperative complications $(P<0.001)$.

\subsection{Univariate Analyses of the Risk Factors of ePOCs}


3.5.1 Demographic and clinical variables. The results of univariate analyses of the risk factors for overall ePOCs in 97 patients undergoing surgery for $C D$ are reported in Table 1. None of the studied demographic variables was significantly associated with the occurrence of postoperative complications. Significant differences $(P<0.001)$ were found in the postoperative complication rate between different disease behavior groups according to Montreal Classification. Higher complication rate $(52.0 \%)$ was observed in penetrating disease compared to non-penetrating disease $(27.8 \%)(P=0.027)$. Patients with multiple lesions had significantly higher percentage of ePOCs (51.4\% vs. $23.3 \%, P=0.005)$.

3.5.2 Medications. Steroid use was associated with a higher incidence of ePOCs (41.2\%), although statistical significance was not reached $(P=0.274)$; similarly, the administration of steroids within 2 months before surgery showed insignificantly higher percentages $(42.1 \%)$ of complications $(P=0.407)$. Infliximab use ( $25 \%$ vs. $34.8 \%, P=0.71)$ and preoperative infliximab exposure $(P=1.00)$ were not risk factors for ePOCs. Analyses concerning other therapeutic regimens revealed no significant differences in ePOC rates.

3.5.3 Laboratory parameters. Patients with serum platelet count $<300(* 1000 / \mathrm{mm} 3)$ had a significant higher rate of ePOCs ( $39.7 \%$ vs. $19.4 \%, P=0.049)$. And there was a significant positive correlation between CRP levels of $>10 \mathrm{~g} / \mathrm{L}$ and ePOCs $(23.6 \%$ vs. $47.6 \%, \mathrm{p}=0.014)$. Other biological variables associated with significantly higher rates of ePOC include prealbumin level $<174 \mathrm{mg} / \mathrm{L}(\mathrm{P}=0.04), \mathrm{GGT}$ level $>10 \mathrm{U} / \mathrm{L}(\mathrm{P}=0.01)$ and serum sodium ion level $<139 \mathrm{mmol} / \mathrm{L}(\mathrm{P}=0.008)$.

3.5.4 Surgical variables. The diagnosis-surgery duration exceeding 6 months is significantly correlated with a higher percentage of ePOCs $(\mathrm{P}<0.001) .7$ patients $(16.3 \%)$ undergoing laparoscopic surgery experienced postoperative complications, compared with 26 patients (48.1\%) undergoing open surgery $(P=0.001)$. There is no significant correlation between the indication for surgery and the occurrence of ePOCs $(\mathrm{P}=0.691)$.

\subsection{Multivariate Analyses of the Risk Factors of ePOCs}

Factors affecting ePOCs incidence at a significance level of $p<0.1$ in univariate analysis were inserted into a binary logistic regression model (Table 3). Diagnosis-surgery duration exceeding 6 months(oddsratio $[\mathrm{OR}]=4.07$; confidence interval $[\mathrm{Cl}] 95 \%[1.10-15.09], \mathrm{P}=0.036)$, serum platelet count $<300 * 1000 / \mathrm{mm}^{3}$ (odds-ratio [OR]=6.74; confidence interval [Cl] 95\%[1.58-28.71], $P=0.01$ ), serum GGT level $>10 \mathrm{U} / \mathrm{L}$ (odds-ratio [OR] $=9.22$; confidence interval [CI] 95\%[1.23-68.99], $\mathrm{P}=0.031$ ) were identified as independent risk factors for the ePOCs. 
Table 1

CD Patients' Baseline Information and univariate analysis of the risk factors for ePOCs ( $\mathrm{n}=97$ )

\section{Clinical Pathological Factors}

Age (yr), m (IQR)

Male, $\mathrm{n}(\%)$

Montreal Classification-Age at diagnosis, $\mathrm{n}(\%)$

A1

A2

A3

Montreal Classification- Location, $\mathrm{n}(\%)$

L1

L2

L3

L4

Montreal Classification-Behavior, n (\%)

B1

B2

B3

Perianal manifestations, $\mathrm{n}(\%)$

Multiple lesions, $\mathrm{n}(\%)$

Previous abdominal surgery, $\mathrm{n}(\%)$

Previous CD-related surgical resection, $\mathrm{n}(\%)$

Previous anal fistula surgery, $\mathrm{n}(\%)$

Previous medication history, n (\%)

Steroids

IFX

5-ASA

Methotrexate

Azathioprine
Value

$43(37-52)$

64(66)

0.918

0.887

2(2.1)

57(58.8)

38(39.2)

0.190

34(35.1)

26(26.8)

36(37.1)

1(1.0)

$<0.001$

22(22.7)

41(42.3)

34(35.0)

0.028

27(27.8)

0.571

37(38.1)

0.005

15(15.5)

0.595

13(13.4)

0.533

13(13.4)

1.000

34(35.1)

0.274

8(8.2)

0.712

41(42.3)

0.982

2(2.1)

0.546

23(23.7)

0.678 


\begin{tabular}{|c|c|c|}
\hline Clinical Pathological Factors & Value & $\begin{array}{l}\mathrm{P} \\
\text { Value }\end{array}$ \\
\hline Tacrolimus & $3(3.1)$ & 0.266 \\
\hline \multicolumn{3}{|l|}{ Preoperative lab index } \\
\hline Leukocytes $\left(/ \mathrm{mm}^{3}\right), \mathrm{m}$ (IQR) & $7.39(5.1-12.5)$ & \\
\hline$>10.0, \mathrm{n}(\%)$ & $31(33)$ & 0.407 \\
\hline Hemoglobin, mean (SD) & $112.3(21.66)$ & \\
\hline$<110.0, \mathrm{n}(\%)$ & $40(41.2)$ & 0.720 \\
\hline Platelets $\left({ }^{\star} 1000 / \mathrm{mm}^{3}\right), \mathrm{m}(\mathrm{IQR})$ & 245(194.75-340.75) & \\
\hline$<300, \mathrm{n}(\%)$ & $63(64.9)$ & 0.049 \\
\hline C-reactive protein $(\mathrm{g} / \mathrm{L}), \mathrm{m}(\mathrm{IQR})$ & 6.38(1.73-21.30) & \\
\hline$>10$ & $42(43.3)$ & 0.014 \\
\hline $\operatorname{ESR}(\mathrm{mm} / \mathrm{h}), \mathrm{m}(\mathrm{IQR})$ & $17(10.25-37.5)$ & \\
\hline$>10$ & $36(75)$ & 1.000 \\
\hline Prealbumin(mg/L),m(IQR) & $\begin{array}{l}155.85(105.73- \\
221.75)\end{array}$ & \\
\hline$<174$ & $48(53.3)$ & 0.040 \\
\hline Albumin(g/L), m(IQR) & $35.2(31.05-40)$ & \\
\hline$<30$ & 19(21.3) & 0.569 \\
\hline $\operatorname{ALT}(\mathrm{U} / \mathrm{L}), \mathrm{m}(\mathrm{IQR})$ & $11(7.0-18.3)$ & \\
\hline$>30$ & $38.5)$ & 0.720 \\
\hline AST (U/L), m(IQR) & $14(11.0-17.25)$ & \\
\hline$>20$ & $16(17.8)$ & 0.618 \\
\hline GGT (U/L), m(IQR) & 17.3(10.45-32.25) & \\
\hline$>10$ & $68(76.4)$ & 0.010 \\
\hline Direct bilirubin (umol/L), m(IQR) & $3.9(2.4-5.12)$ & \\
\hline$>2.4$ & $65(72.2)$ & 0.219 \\
\hline Total bilirubin (umol/L), m(IQR) & $9.6(5.5-12.7)$ & \\
\hline$>12$ & $23(25.3)$ & 0.541 \\
\hline Urea (mmol/L), m(IQR) & $4.4(3.07-5.7)$ & \\
\hline
\end{tabular}




\begin{tabular}{|c|c|c|}
\hline Clinical Pathological Factors & Value & $\begin{array}{l}\mathrm{P} \\
\text { Value }\end{array}$ \\
\hline$>57$ & $22(24.7)$ & 0.930 \\
\hline Creatinine (umol/L), m(IQR) median (IQR) & $59.1(47.5-71)$ & \\
\hline$<47$ & $22(24.7)$ & 0.138 \\
\hline Uric acid(umol/L), m(IQR) & 216.45(96.49) & \\
\hline$<150$ & 23(25.8) & 0.794 \\
\hline $\mathrm{Na}+(\mathrm{mmol} / \mathrm{L}), \mathrm{m}(\mathrm{IQR})$ & $139(137-141)$ & \\
\hline$<139$ & $49(51.6)$ & 0.008 \\
\hline $\mathrm{K}+(\mathrm{mmol} / \mathrm{L}), \mathrm{m}(\mathrm{IQR})$ & $3.9(3.4-4.2)$ & \\
\hline$<3.5$ & $32(33.7)$ & 0.796 \\
\hline Procalcitonin(ng/ml), m(IQR) & $0.06(0.02-2.97)$ & \\
\hline$>0.02$ & $22(75.9)$ & 0.646 \\
\hline \multicolumn{3}{|c|}{$\begin{array}{l}\text { Preoperative medical treatment (within } 2 \text { months before surgery), } \\
\mathrm{n}(\%)\end{array}$} \\
\hline Steroids & 19(19.6) & 0.407 \\
\hline IFX & $1(1)$ & 1.000 \\
\hline 5-ASA & $26(26.8)$ & 0.940 \\
\hline Methotrexate & $9(9.3)$ & 0.714 \\
\hline Azathioprine & $0(0)$ & प \\
\hline Tacrolimus & $1(1)$ & 1.000 \\
\hline Diagnosis-surgery duration (months), m(IQR) & $4(0-25.5)$ & \\
\hline$>6, n(\%)$ & $40(41.2)$ & $<0.001$ \\
\hline Emergency operation, $n(\%)$ & $23(23.7)$ & 0.273 \\
\hline Laparoscopic surgery, n(\%) & $43(44.3)$ & 0.001 \\
\hline Anastomosis, $\mathrm{n}(\%)$ & $90(92.8)$ & 1.000 \\
\hline Indications for surgery, n(\%) & & 0.691 \\
\hline Obstruction & $40(41.2)$ & \\
\hline Fistula & 10(10.3) & \\
\hline Perforation & 18(18.6) & \\
\hline
\end{tabular}




\begin{tabular}{|lll|}
\hline Clinical Pathological Factors & Value & Palue \\
\hline Bleeding & $6(6.2)$ & \\
\hline Medication- refractory diseases & $5(5.2)$ & \\
\hline Others & $18(18.6)$ & $<0.001$ \\
\hline Length of stay in hospitals (days), m(IQR) & $10(7-13)$ & \\
\hline
\end{tabular}


Table 2

ePOCs in 97 postoperative CD patients

\begin{tabular}{|ll|}
\hline & $\mathrm{n}(\%)$ \\
\hline Number of patients having ePOCs & $33(34.0)$ \\
\hline ePOC cases $(n)$ & 42 \\
\hline Multiple complications & $6(6.2)$ \\
\hline Intraabdominal septic complications & $11(11.3)$ \\
\hline Abdominal abscess & $9(9.3)$ \\
\hline Anastomotic leak & $2(2.1)$ \\
\hline Extra-abdominal septic complications & $19(19.6)$ \\
\hline Wound infection & $13(13.4)$ \\
\hline Pneumonia & $5(5.2)$ \\
\hline Septic shock & $1(1.0)$ \\
\hline Non-septic complications & $12(12.4)$ \\
\hline Enterocutaneous fistula & $1(1.0)$ \\
\hline Bleeding & $1(1.0)$ \\
\hline Bowel obstruction & $5(5.2)$ \\
\hline Gastric retention & $2(2.1)$ \\
\hline Thrombosis & 0 \\
\hline Malnutrition & $1(1.0)$ \\
\hline Anemia & $1(1.0)$ \\
\hline Death & $1(1.0)$ \\
\hline Clavien- Dindo Classification & \\
\hline Mild complications & $25(25.8)$ \\
\hline Grade I & $7(7.2)$ \\
\hline Grade II & $18.2)$ \\
\hline Severe complications & \\
\hline Grade III & \\
\hline
\end{tabular}




\begin{tabular}{|lc|}
\hline & $\mathrm{n}(\%)$ \\
\hline Grade $V$ & $1(1.0)$ \\
\hline
\end{tabular}

Page $12 / 19$ 
Table 3

Multivariate Analysis of the Risk Factors of ePOCs ( $n=97)$

\begin{tabular}{|c|c|c|}
\hline Risk Factors & Odds ratio $(95 \% \mathrm{Cl})$ & P Value \\
\hline \multicolumn{3}{|l|}{ Multiple lesions } \\
\hline No & Reference Range & \\
\hline Yes & $2.57(0.62-10.61)$ & 0.19 \\
\hline \multicolumn{3}{|l|}{ Clinical disease behavior } \\
\hline Non-penetrating disease & Reference Range & \\
\hline Penetrating disease & $1.44(0.33-6.34)$ & 0.63 \\
\hline \multicolumn{3}{|l|}{ C-reactive protein(g/L) } \\
\hline$<10$ & Reference Range & \\
\hline$>10$ & $3.15(0.78-12.70)$ & 0.11 \\
\hline \multicolumn{3}{|l|}{ Platelet count $\left(* 1000 / \mathrm{mm}^{3}\right)$} \\
\hline$>300$ & Reference Range & \\
\hline$<300$ & $6.74(1.58-28.71)$ & 0.01 \\
\hline \multicolumn{3}{|l|}{$\mathrm{GGT}(\mathrm{U} / \mathrm{L})$} \\
\hline$<10$ & Reference Range & \\
\hline$>10$ & $9.22(1.23-68.99)$ & 0.03 \\
\hline \multicolumn{3}{|l|}{$\mathrm{Na}+(\mathrm{mmol} / \mathrm{L})$} \\
\hline$<139$ & Reference Range & \\
\hline$>139$ & $0.41(0.11-1.59)$ & 0.20 \\
\hline \multicolumn{3}{|c|}{ Diagnosis- surgery duration(months) } \\
\hline$<6$ months & Reference Range & \\
\hline$>6$ months & $4.07(1.10-15.09)$ & 0.04 \\
\hline \multicolumn{3}{|l|}{ Surgical approach } \\
\hline Laparotomy & Reference Range & \\
\hline Laparoscopic surgery & $0.34(0.86-1.37)$ & 0.34 \\
\hline
\end{tabular}

\section{Discussion}


Surgical resection for CD patients is correlated to high ePOC risks. And some serious postoperative complications such as intra-abdominal septic complications occur more frequently in $C D$ than other diseases ${ }^{16}$, necessitating evaluation of the frequency and risk factors of ePOCs for CD.

In this retrospective study, 34.0\% patients experienced at least one $\mathrm{ePOC}$, wound infection being the most frequent complication. This incidence is comparable with the current literature, ranging from $21-37 \%{ }^{7-}$ 13. The mortality rate was $1.0 \%$ and IASCs were recorded in $11.3 \%$ cases, in line with previous studies ${ }^{8-12}$. Numerous studies examined the incidence of ePOCs. A retrospective multi-center study $(n=231)$ of an adult cohort in three countries (Japan, Brazil and Italy) reported an overall ePOCs of $24 \%$. Among 55 reported complications, $12 \%$ were intra-abdominal septic complications including anastomotic leakage (8\%) and intra-abdominal abscesses (4\%). Blood transfusion and perforating disease were independent significant risk factors for overall complications and IASCs, respectively ${ }^{8}$. Another more recent retrospective study in Europe evaluated the risk of postoperative complications in 199 CD patients. 62 (31\%) patients experienced at least some kind of ePOCs and IASCs occurred in 34 patients (17\%). One patient $(0.5 \%)$ died from septic shock following proctocolectomy. A bivariate analysis revealed that surgical anastomosis and preoperative hemoglobin level of $<10 \mathrm{~g} / \mathrm{dl}$ were associated with an increased postoperative IASC rate ${ }^{11}$.

Due to high rates of ePOCs for CD surgeries, preoperative risk evaluation of complications seems pivotal to patient optimization for surgery ${ }^{17}$. In our study, A diagnosis-surgery duration $>6$ months(odds-ratio $[\mathrm{OR}]=4.07$; confidence interval $[\mathrm{Cl}] 95 \%[1.10-15.09], \mathrm{P}=0.036)$, serum platelet count $<300 * 1000 / \mathrm{mm}^{3}$ (odds-ratio [OR] $=6.74$; confidence interval [Cl] 95\%[1.58-28.71], $P=0.01$ ), serum GGT level $>10 \mathrm{U} / \mathrm{L}$ (odds-ratio [OR] $=9.22$; confidence interval [CI] 95\%[1.23-68.99], $\mathrm{P}=0.031$ ) were significantly associated with a higher risk for ePOCs in a binary regression.

As a progressive disease, $C D$ results in cumulative bowel damage over time, leading to irreversible complications $^{18}$. In a population-based study on the CD progression, about $19 \%$ of patients had already experienced penetrating or stricturing complications within the first 3 months of diagnosis, but complications occurred in $50 \%$ of patients within 20 years after diagnosis ${ }^{19}$, indicating longer disease duration is associated with increasing disease severity. Therefore, rapid and early intervention including medications and surgery may effectively prevent CD progression so as to reduce the risk of adverse complications and healthcare burden ${ }^{20}$. Shorter disease duration is related to a higher response rate to biologic agents in $C D$ patients ${ }^{21}$. Issues regarding on the safety of early surgical intervention for $C D$ patients, however, are still a subject to debate without a clear consensus. Recently published data from a retrospective study with $153 \mathrm{CD}$ patients undergoing elective ileocolic resection in a Greek tertiary center revealed that disease duration was not an independent predictor for POCs: complication rates in those who went on surgery $<5$ years, in 5-10 years and $>10$ years after diagnosis were $16.1 \%, 13.9 \%$ and $36.4 \%$, respectively, with no intergroup differences in the incidence of POCs ${ }^{12}$. Our series, however, reported a significant correlation between diagnosis-surgery duration $>6$ months and ePOCs, in accordance with some previous large studies. For example, a retrospective study in France, concerning 592 consecutive 
patients who underwent surgery for $C D$, reported that $C D$ duration $>2$ years is significantly associated with ePOCs $(P=0.01)$ in univariate analysis ${ }^{10}$. We believe that our findings augment the evidence for the negative effect of long diagnosis-surgery duration on the postoperative course of CD patients. Therefore, we recommend early surgery for eligible patients in order to avoid postoperative complications. Elżbieta et al. examined the relationship between CD duration and CARD15 expression, one of the susceptibility genes in CD, and found a significantly higher peripheral mRNA level of the CARD15 in patients with disease duration between 12 and 60 months, compared to patients whose disease duration was $<12$ months ${ }^{22}$. We speculated that higher CARD15 expression over longer disease duration modified the disease behavior ${ }^{23}$, making it more complicated, thus leading to a higher rate of ePOCs. And further studies are needed to precisely explore the safety of early surgical intervention and its ability to prevent disease progression in $\mathrm{CD}$.

It is important to understand how modifiable factors, especially pre-operative lab parameters, affect outcomes after surgical interventions, so that we can identify high-risk CD population for preoperative optimization. Current data concerning these aspects are, however, relatively scarce, focusing mainly on hemoglobin, CRP and albumin level 9,10,12. Therefore, one of the unique aspects of our study is the evaluation of numerous possible preoperative lab data. In multivariate analysis, serum platelet count $<300 * 1000 / \mathrm{mm}^{3}$ and serum GGT level $>10 \mathrm{U} / \mathrm{L}$ were found to be independent risk factors of ePOCs. Low platelet counts can serve as an indicator for active $\mathrm{CD}^{24}$, which also helps to explain the correlation between low serum platelet count and a higher risk of ePOCs. On the other hand, Jessika et al. ${ }^{25}$ determined that the presence of liver test abnormalities, including gamma-glutamyl transpeptidase, was identified as an indicator for complicated $\operatorname{CD}(\mathrm{HR} 2.6, \mathrm{p}<0.0001)$. And elevated gamma-glutamyl transpeptidase itself suggests abnormal liver function, which might account for a higher risk of ePOCs. Therefore, according to our study, CD patients who are going to be operated but with serum platelet count $<300 * 1000 / \mathrm{mm}^{3}$ or gamma-glutamyl transpeptidase level $>10 \mathrm{U} / \mathrm{L}$ can be better prepared. Abnormal lab values need to be corrected in these patients to improve postoperative safety.

Anti-TNF-alpha agents have been used in the treatment of CD, and their efficacy on CD progression has been well demonstrated ${ }^{3-5}$. Early initiation of infliximab within the first 2 years of diagnosis reduces the rate of surgery ${ }^{26}$. However, given its potential impact on wound healing and immunosuppressive properties, a crucial concern is whether patients undergoing major abdominal surgery after anti-TNF drug exposure are at increased risk of early postoperative complications ${ }^{27}$. In our series, previous exposure to anti-TNF agents in CD patients was not a risk factor for ePOCs, in line with most studies. A Mayo Clinic study ${ }^{28}$ supported this idea by illustrating no association between infliximab use and early complications after abdominal surgery for CD. A Los Angeles study ${ }^{27}$ revealed a higher but statistically insignificant rate of adverse outcomes in anti-TNF detectable versus undetectable group. Another Canadian retrospective case-control study ${ }^{29}$ compared postoperative outcomes of $C D$ patients with or without exposure to antiTNF agents within 180 days of abdominal surgery, but no intergroup difference was found. The most recent, prospective multicentric literature that included serum drug level measures at time of surgery 
found no association between preoperative exposure to anti-TNF and postoperative complications ${ }^{30}$. Some studies, however, reached contrary conclusions. Antoine et al. ${ }^{10}$ in 2018 found that preoperative anti-TNF therapy was a predictor of morbidity after surgery for ileocolonic CD. Some meta-analyses indicated that preoperative anti-TNF exposure was a risk factor of POCs in CD patients ${ }^{31-34}$. Most of the studies have been criticized for not being able to control crucial confounding factors, such as disease severity, nutritional status as well as preoperative corticosteroid use, which make conclusions less convincing: elevated postoperative complication rates may just be a reflection of disease severity and a higher likelihood of poor nutritional status, but not a direct effect of preoperative anti-TNF exposure. More valid data are needed to confirm our results.

Our study presents certain limitations. First, the retrospective design indicates that data can only be passively retrieved from patients' medical records. Therefore, some data are incomplete or even not collected at all, including procalcitonin levels, configuration of anastomosis (end-to-end vs. side- to-side), patients' smoking status, BMI values, which are presumable risk factors for ePOCs. Secondly, the sample size $(n=97)$ is not big enough due to the single-center design, which may lead to insufficient size of some subgroups (such as patients with anti-TNF preoperative exposure). Thus, detection of differences may be more difficult and some risk factors for ePOCs might be precluded. Finally, we choose patients from a high-level center that managed CD patients with complicated and severe conditions, suggesting poorer prognosis than other patients despite well-trained and experienced surgeons and multidisciplinary teams. However, we believe that our study adds more new and convincing evidence of risk factors of ePOCs in CD patients.

In conclusion, the incidence of ePOCs was $34.0 \%$ among CD patients undergoing surgical resection. Diagnosis-surgery duration exceeding 6 months, serum platelet count $<300 * 1000 / \mathrm{mm}^{3}$ and serum GGT level $>10 \mathrm{U} / \mathrm{L}$ were identified as independent risk factors for the ePOCs. And preoperative anti-TNF exposure was not associated with an elevated ePOC rate.

\section{Conclusions}

- Overall, we summarized that the diagnosis-surgery duration exceeding 6 months, serum platelet count $<300 * 1000 / \mathrm{mm}^{3}$, and serum GGT level $>10 \mathrm{U} / \mathrm{L}$ were associated with an increased risk of ePOCs. Preoperative exposure to anti-TNF agents were not associated with a higher risk of ePOCs. As physicians, the interest of the patient should always be well considered, by reducing the diagnosis-surgery duration, which means to get surgery done sooner, will significantly reduce the early postoperative complications, pain and financial burden of the patient.

\section{Declarations}

Ethics approval and consent to participate: All methods were carried out in accordance with relevant guidelines and regulations. All experimental protocols were approved by the Ethics Committee of 
Shanghai Jiao Tong University School of Medicine Affiliated Renji Hospital. Informed consents were obtained from all subjects involved in this manuscript.

Consent for publication: Not applicable

Availability of data and materials: The datasets used and/or analysed during the current study are available from the corresponding author on reasonable request. The data are not publicly available due to some containing information that could compromise participant privacy.

Competing interests: The authors declare that they have no competing interests.

Funding: The work was supported by the Cultivation Clinical Research Grants from Renji Hospital affiliated to Shanghai Jiao Tong University School of Medicine (PYIII20-14 to Yumin Hao).

Author contributions: XC and YM participated in the conception, design, drafting of the study protocol, recruitment of participants. SZ, SL and YJ were responsible for data acquisition. XC and SZ participated in the analysis and interpretation of the data. FS, YS and YQ contributed to the revising of the paper. YM and $\mathrm{MZ}$ were responsible for the final approval of the version to be published. And all authors agree to be accountable for all aspects of the work.

Acknowledgements: Not applicable

\section{References}

1. Roda G, Chien Ng S, Kotze PG, et al. Crohn's disease. Nat Rev Dis Primers 2020; 6(1): 22.

2. Molodecky NA, Soon IS, Rabi DM, et al. Increasing incidence and prevalence of the inflammatory bowel diseases with time, based on systematic review. Gastroenterology 2012; 142(1): 46-54 e42; quiz e30.

3. Colombel JF, Sandborn WJ, Reinisch W, et al. Infliximab, azathioprine, or combination therapy for Crohn's disease. N Engl J Med 2010; 362(15): 1383-95.

4. Adegbola SO, Sahnan K, Warusavitarne J, Hart A, Tozer P. Anti-TNF Therapy in Crohn's Disease. Int J Mol Sci 2018; 19(8).

5. Argollo M, Fiorino G, Peyrin-Biroulet L, Danese S. Vedolizumab for the treatment of Crohn's disease. Expert Rev Clin Immunol 2018; 14(3): 179-89.

6. Frolkis AD, Dykeman J, Negron ME, et al. Risk of surgery for inflammatory bowel diseases has decreased over time: a systematic review and meta-analysis of population-based studies. Gastroenterology 2013; 145(5): 996-1006.

7. Biroulet LP, Loftus EV, Harmsen WS, et al. 490 Postoperative Complications in a Population-Based Cohort of Crohn's Disease. Gastroenterology 2010; 138(5, Supplement 1): S-70.

8. Yamamoto T, Spinelli A, Suzuki Y, et al. Risk factors for complications after ileocolonic resection for Crohn's disease with a major focus on the impact of preoperative immunosuppressive and biologic 
therapy: A retrospective international multicentre study. United European Gastroenterol J 2016; 4(6): 784-93.

9. Fumery M, Seksik P, Auzolle C, et al. Postoperative Complications after lleocecal Resection in Crohn's Disease: A Prospective Study From the REMIND Group. Am J Gastroentero/ 2017; 112(2): 337-45.

10. Brouquet A, Maggiori L, Zerbib P, et al. Anti-TNF Therapy Is Associated With an Increased Risk of Postoperative Morbidity After Surgery for lleocolonic Crohn Disease: Results of a Prospective Nationwide Cohort. Ann Surg 2018; 267(2): 221-8.

11. lesalnieks I, Spinelli A, Frasson M, et al. Risk of postoperative morbidity in patients having bowel resection for colonic Crohn's disease. Tech Coloproctol 2018; 22(12): 947-53.

12. Gklavas A, Poulaki A, Dellaportas D, Papaconstantinou I. Risk factors for postoperative complications after elective ileocolic resection for Crohn's disease: a retrospective study. Ann Gastroenterol 2020; 33(6): 645-55.

13. Kulaylat AN, Kulaylat AS, Schaefer EW, et al. The Impact of Preoperative Anti-TNFa Therapy on Postoperative Outcomes Following lleocolectomy in Crohn's Disease. J Gastrointest Surg 2021; 25(2): 467-74.

14. Silverberg MS, Satsangi J, Ahmad T, et al. Toward an integrated clinical, molecular and serological classification of inflammatory bowel disease: report of a Working Party of the 2005 Montreal World Congress of Gastroenterology. Can J Gastroenterol 2005; 19 Suppl A: 5a-36a.

15. Dindo D, Demartines N, Clavien PA. Classification of surgical complications: a new proposal with evaluation in a cohort of 6336 patients and results of a survey. Ann Surg 2004; 240(2): 205-13.

16. Lipska MA, Bissett IP, Parry BR, Merrie AE. Anastomotic leakage after lower gastrointestinal anastomosis: men are at a higher risk. ANZ J Surg 2006; 76(7): 579-85.

17. Spinelli A, Allocca M, Jovani M, Danese S. Review article: optimal preparation for surgery in Crohn's disease. Aliment Pharmacol Ther 2014; 40(9): 1009-22.

18. Pariente B, Mary JY, Danese $S$, et al. Development of the Lémann index to assess digestive tract damage in patients with Crohn's disease. Gastroenterology 2015; 148(1): 52-63.e3.

19. Thia KT, Sandborn WJ, Harmsen WS, Zinsmeister AR, Loftus EV. Risk Factors Associated With Progression to Intestinal Complications of Crohn's Disease in a Population-Based Cohort. Gastroenterology 2010; 139(4): 1147-55.

20. Danese S, Fiorino G, Peyrin-Biroulet L. Early intervention in Crohn's disease: towards disease modification trials. Gut 2017; 66(12): 2179.

21. Faleck DM, Winters A, Chablaney S, et al. Shorter Disease Duration Is Associated With Higher Rates of Response to Vedolizumab in Patients With Crohn's Disease But Not Ulcerative Colitis. Clinical Gastroenterology and Hepatology 2019; 17(12): 2497-505.e1.

22. Poniewierka E, Neubauer K, Kempiński R, Sadakierska-Chudy A. Disease duration and age influence CARD15 expression in Crohn's disease. Postepy Hig Med Dosw (Online) 2016; 70: 10-3. 
23. Tsianos EV, Katsanos KH, Tsianos VE. Role of genetics in the diagnosis and prognosis of Crohn's disease. World J Gastroenterol 2012; 18(2): 105-18.

24. Öztürk ZA, Dag MS, Kuyumcu ME, et al. Could platelet indices be new biomarkers for inflammatory bowel diseases? Eur Rev Med Pharmacol Sci 2013; 17(3): 334-41.

25. Barendregt J, de Jong M, Haans JJ, et al. Liver test abnormalities predict complicated disease behaviour in patients with newly diagnosed Crohn's disease. Int J Colorectal Dis 2017; 32(4): 459-67.

26. Ma C, Beilman CL, Huang VW, et al. Anti-TNF Therapy Within 2 Years of Crohn's Disease Diagnosis Improves Patient Outcomes: A Retrospective Cohort Study. Inflamm Bowel Dis 2016; 22(4): 870-9.

27. Lau C, Dubinsky M, Melmed G, et al. The impact of preoperative serum anti-TNFa therapy levels on early postoperative outcomes in inflammatory bowel disease surgery. Ann Surg 2015; 261(3): 487-96.

28. Colombel JF, Loftus EV, Tremaine WJ, et al. Early postoperative complications are not increased in patients with Crohn's disease treated perioperatively with infliximab or immunosuppressive therapy. Am J Gastroenterol 2004; 99(5): 878-83.

29. Waterman M, Xu W, Dinani A, et al. Preoperative biological therapy and short-term outcomes of abdominal surgery in patients with inflammatory bowel disease. Gut 2013; 62(3): 387-94.

30. Adamina M, Fiorino G. At the Crossroads of Caution and Intervention: Anti-TNF Therapy Prior to Elective CD Surgery. J Crohns Colitis 2021; 15(10): 1778-9.

31. Billioud V, Ford AC, Tedesco ED, Colombel JF, Roblin X, Peyrin-Biroulet L. Preoperative use of anti-TNF therapy and postoperative complications in inflammatory bowel diseases: a meta-analysis. $J$ Crohns Colitis 2013; 7(11): 853-67.

32. Yang ZP, Hong L, Wu Q, Wu KC, Fan DM. Preoperative infliximab use and postoperative complications in Crohn's disease: a systematic review and meta-analysis. Int J Surg 2014; 12(3): 22430.

33. Waterland P, Athanasiou T, Patel H. Post-operative abdominal complications in Crohn's disease in the biological era: Systematic review and meta-analysis. World J Gastrointest Surg 2016; 8(3): 274-83.

34. Marchal L, D'Haens G, Van Assche G, et al. The risk of post-operative complications associated with infliximab therapy for Crohn's disease: a controlled cohort study. Aliment Pharmacol Ther 2004; 19(7): 749-54. 